\title{
A NEW SUSPENSION SYSTEM OF AN AUTONOMOUS CATERPILLAR PLATFORM
}

\author{
ANDRZEJ JURKIEWICZ \\ AGH University of Science and Technology, Faculty of Mechanical Engineering and Robotics, Kraków, Poland \\ e-mail: jurkand@agh.edu.pl \\ TOMASZ NABAGŁO \\ Cracow University of Technology, Faculty of Mechanical Engineering, Kraków, Poland \\ e-mail: tnabaglo@mech.pk.edu.pl \\ Janusz Kowal, Marcin Apostol \\ AGH University of Science and Technology, Faculty of Mechanical Engineering and Robotics, Kraków, Poland \\ e-mail: jkowal@agh.edu.pl; apostol@agh.edu.pl
}

\begin{abstract}
The article contains results of a conceptual work and research which develops a semi-active suspension of the 2S1 caterpillar platform. The results are collected on designed and built test stands and used in three alternative models of the platform suspension system. The first one is based on the existing construction of the platform. The other two have been modernized by using new solutions in the tracked vehicles suspension technology associated with a torsional spring element. The authors assumed simulation conditions for straight line driving while the model overcomes an obstacle in one track. The results of simulations are compared and analyzed to improve parameters of the vehicle suspension.
\end{abstract}

Keywords: caterpillar platform, test stand, logarithmic spring, simulation, ADAMS

\section{Introduction}

The paper presents results of a conceptual work and research which has been carried out within the Development Project titled "Autonomous, universal caterpillar platform for combat and logistics tasks according to standards of the modern battlefield". The authors describe there, laboratory researches, which give a set of data. These data have been used in simulation tests in next part of the article. Kowal et al. (2013) described a new type of the suspension system for a caterpillar platform, but also they described a test stand for measurement of static characteristics of suspension springs. The test stand for measurement of dynamic characteristics of spring elements was more precisely put forward by Jurkiewicz et al. (2010). These characteristics may be verified with the Finite Element Method (FEM) model, what was realized also by Kowal et al. (2013). They took into consideration two types of torsional springs. One of them is the so called torsional spring based on the Archimedes curve, and the second one is the so called torsional spring based on the logarithmic curve. The authors of this article compared such parameters like total deformation of springs and stresses inside the springs. They also presented static characteristics of the above mentioned types of torsional springs. This is the base of the next part of our article. In the next part of the article, the three versions of the tracked vehicle suspension model have been described. The first one is the basic model, based on a torsional bars system. The construction process of this model and also the simulation of the model was described by Nabagło et al. (2011). They described also modified models of the tracked vehicle (Nabagło et al., 2014). The second model is the so called first modernized model, where the torsional bars in the second axle have been replaced with spiral springs. And finally, the last 
model is presented. In this model, all torsional bars are replaced with spiral springs. In the next sections, it is called the second modernized model. As has been mentioned, during researches, the suspension system of $2 \mathrm{~S} 1$ tracked vehicle has been modernized by usage of logarithmic springs. But not only by this. In these two above modernized models also other features have been changed. The second element has been an idler mechanism. This mechanism has been replaced with a constant force actuator which acts on the idler wheel. This solution enables one to hold a constant value of track tension. In the basic model, the idler mechanism has been blocked in a fixed position. Ryu et al. (2000) described more detailed rules and parameters of connection links in the case of two links of a track. From the mathematical point of view, the same solution has been used in all the three mentioned suspension models. In these models, contact forces act between a single track link and a single wheel and also between a single track link and the ground. Contact forces, which have been applied between the wheel and ground, were described by Lee (2000). Specific parameters of these connections and also parameters of the forces have been described in ADAMS (ADAMS/View, Solver Documentation, 2010). In the simulation process, the authors used sometimes exact geometry of theelements. For this reason, during model construction, designers have to solve a multi-body CAD designing problem, what was put forward by Łukaszewicz (2009). In continuation of the simulation topic, Kubela et al. (2010) described a multi-body mechanism simulation in ADAMS. They showed the co-simulation environment with a Matlab-Simulink program. Such a solution could be applied for calculation of more complicated control tasks or nonlinear elements reactions in the Simulink program. Huh et al. (2011) took under consideration a specific idler mechanism for the track tension control. The simulations and also measurements on a real tracked vehicle were conducted in conditions when the vehicle turned on a flat road. In contrast to Huh et al. (2011) article, in this paper, in the case of the modernized tacked vehicle, the authors also describe the track tension mechanism, but modeled with a single force acting in the longitudinal axle of the vehicle. Also the driving conditions are different, because the authors assume the straight line driving on a flat road with a single obstacle. For finding all the above mentioned vehicle solutions, the authors use an indicator which shows the absorbed power by a human body. This indicator is based on the human body power absorption in the vertical direction, which was described by Lee and Lins (1973). This work is associated with the model developed by the U.S. Army Tank-Automotive Research and Development Center (TARDEC). This model treats a human body as a filter of the vibrations power which is absorbed by it, because the human body reacts with different sensitivity to different vibration frequencies. According to that work, to hold a comfort feeling, the power absorption of the human body must not exceed 6 watts, what was described by Donahue and Hedrick (2003).

\section{Caterpillar platform suspension modernization}

Modernization of the caterpillar platform has been associated with torsional bars replaced with torsional springs in the chosen axis of the suspension system. The research revealed that the usage of the so-called logarithmic springs is the best solution here. Repeatable units of the suspension based on torsion bars or torsional springs are presented in Fig. 1. In Fig. 1b, a packet of torsional springs consists of 15 parallel connected spiral springs. They are enclosed inside a so-called drum.

An advantage of this solution is associated with using less space, because with torsional springs the whole space under the crew cabin is free. Reactive elements of the suspension system by this way are mounted to the left and right side of the vehicle. To keep the same dynamic parameters of the whole platform, the torsional springs should have static and dynamic properties as close as possible to the static and dynamic properties of the torsional bars. The springs 
(a)

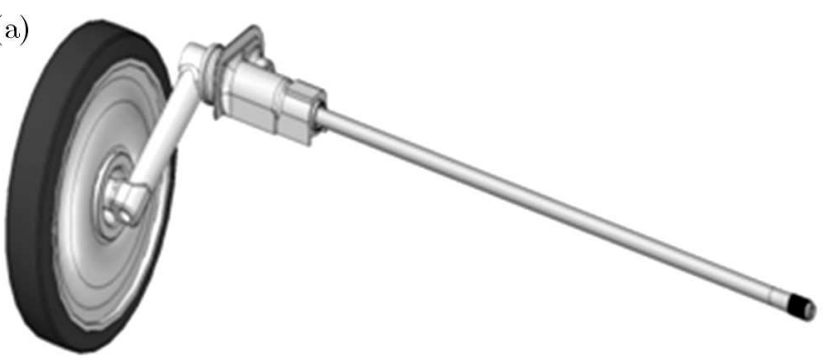

(b)

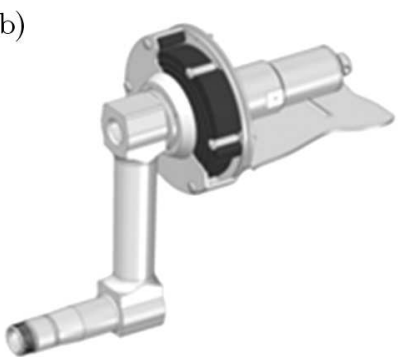

Fig. 1. Elements of the of suspension: (a) with torsion bar, (b) with logarithmic springs packet

packet enclosed in the drum gives some additional possibilities. To the drum, the pressured oil may be pumped, and this way, the torsional spring system properties may be changed.

\section{Test stand researches}

Before starting the tests on the stand, the authors conducted many simulations of stress and strength of the material on a model which has been based on the Finite Element Method (FEM). This model enables determination of the limits of such factor as the suspension arm displacement (see Fig. 2). This model has been also used for verification of static characteristics of the logarithmic spring. One of these characteristics is used in further simulations of the caterpillar platform.

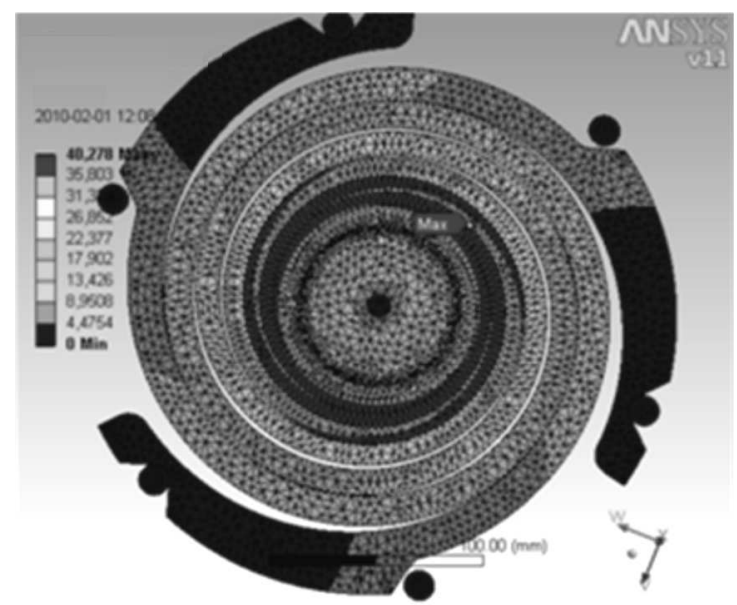

Fig. 2. Total deformation of a spring with a logarithmic curve profile

The main aim of the conducted tests has been connected with finding a torsional spring solution whose static characteristics would be nearest to those of the torsional bar. As the pattern, the static characteristics of the torsional bars installed in the basic version of the caterpillar platform have been assumed. The next step has been associated with investigation of dynamic characteristics chosen according to the above mentioned criteria.

\subsection{Static characteristics of the logarithmic spring}

The measurement and study of static characteristics has been associated with determination of the suspension characteristics for a static force as function of the vertical deflection. The study has been conducted on a group of static characteristics, where the parameter is the oil pressure in the suspension drum. The range of changes encloses between 0 to 20 bar. A force element is 
the actuator. The movement range of its cylinder has been enclosed between 0 to $250 \mathrm{~mm}$. The suspension reaction force has been continuously recorded by summation of the two signals from the force sensors (see Fig. 3a). The guide and the bearing enabled minimization of undesirable friction forces which appear in the load system. The ultimate goal of this study is the selection of static characteristics of the springs (see Fig. 3b) that correspond to characteristics of the original torsional bars. It has been found, as a result of that study, that this condition is fulfilled with one of the solutions, known as the logarithmic spring. In addition, such springs have high damping properties, which can be observed on the static characteristics in form of a substantial frictional hysteresis. A characteristic feature of this construction is that the friction hysteresis is only slightly dependent on the oil pressure which supplies the drum. For this reason, this dependency may be omitted in the following discussion.
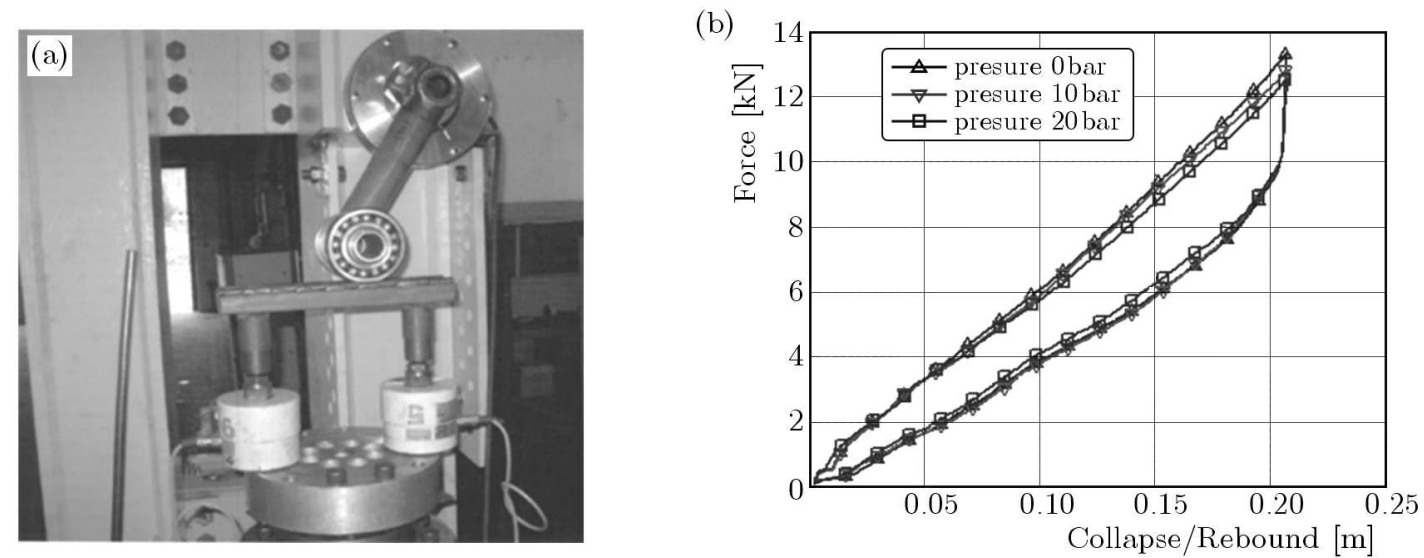

Fig. 3. Measurement system for static examinations (a) and stiffness characteristics of the suspension based on logarithmic springs (b)

\subsection{Dynamic characteristics of the logarithmic spring}

The measurement and study of dynamic characteristics have been conducted on torsional springs whoose static parameters were the most closely related to the characteristics of the original torsional bars. The tests have been carried out on a special test stand where the force actuator is a hydro-pulser. The actuator has been mounted vertically on a supporting structure (see Fig. 4a). The tested suspension has been connected through the ball joint and the force sensor with a hydro-pulser rod. The suspension has been preloaded with a mass element. The mass has been chosen to set up static deflection on the level corresponding to the middle point of the static characteristics deflection. The designed and built test stand enabled measuring dynamic characteristics of the suspension in function of frequency. During investigation, the authors assumed the frequency range from 0 to $10 \mathrm{~Hz}$. Amplitude range has been assumed from -10 to $10 \mathrm{~mm}$. However, the oil pressure range of the suspension actuator has been assumed in the range from 0 to 20 bar. The aim of the study has been connected with determination of the characteristics set of the amplitude-frequency response. During the tests, the hydro-pulser piston displacement $\left(A_{1}\right)$ and vertical displacement of the load $\left(A_{2}\right)$ have been recorded. The amplitude-frequency response is expressed with formula Transmissibility $=A_{2} / A_{1}$. It is presented on the characteristics curves as a frequency function (see Fig. 4b). The characteristics have been measured for various values of the oil pressure in the suspension drum.

The study of dynamic characteristics shows that the chosen spring has the natural frequency, whoose value equals $2.5 \mathrm{~Hz}$, when the above mentioned load system is assumed. The dependency of the amplitude-frequency response as reaction to the oil pressure level is low. For this reason, this dependency can be omitted, similarly like in the case of the static characteristics. 

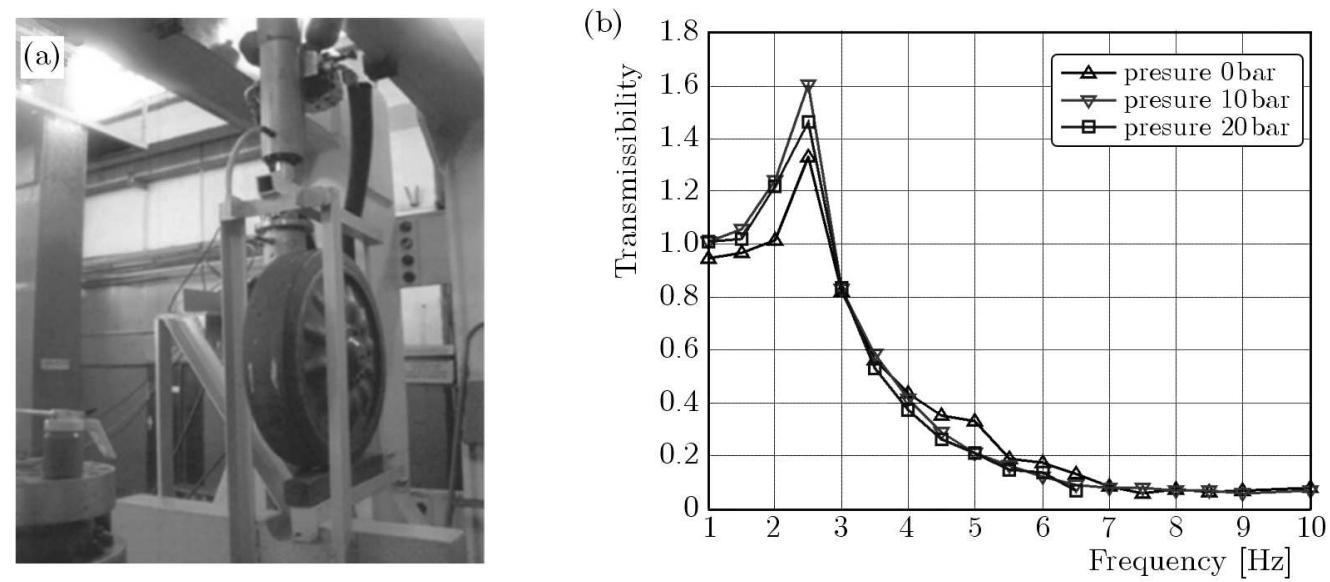

Fig. 4. Measurement system for dynamic investigations (a) and transmissibility characteristics of the suspension based on logarithmic springs (b)

\section{The modeling of the caterpillar platform solutions}

In the paper three solutions of the tracked vehicle suspension model are taken under consideration. The first one is the so called basic model. Its suspension system is based on torsional bars. The second model is modernized with usage of two logarithmic torsional springs, which are mounted on the second axle. The third suspension model is based on torsional springs in all axles. In all of these three models, dampers are mounted at the first and last road wheel axles (see Figs. 7 and 8). The damping characteristics assumed for the dampers, were more precisely described by Nabagło et al. (2011) and shown in Fig. 5.

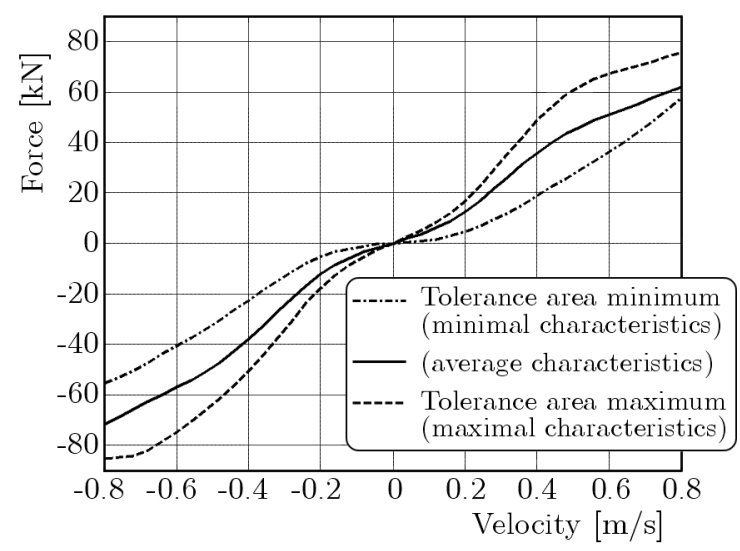

Fig. 5. Damping characteristics tolerance area

The characteristics of the set of 15 logarithmic torsional springs have been calculated based on chosen stiffness characteristics (see Fig. 4a). Because the influence of the oil pressure in the suspension drum may be omitted, the authors examined stiffness characteristics for the torsional springs under no pressure. Because the above characteristics have been measured for the translational system, the stiffness characteristics for the rotational system had to be calculated. The results of that calculation are presented in form of stiffness characteristics of the 15-spring packet in Fig. 6b. In Fig. 6a, an original shape of the logarithmic torsional spring is presented.

The stiffness characteristics presented in Fig. $6 \mathrm{~b}$ are used in both modernized models for description of stiffness properties of the logarithmic torsional springs. 
(a)

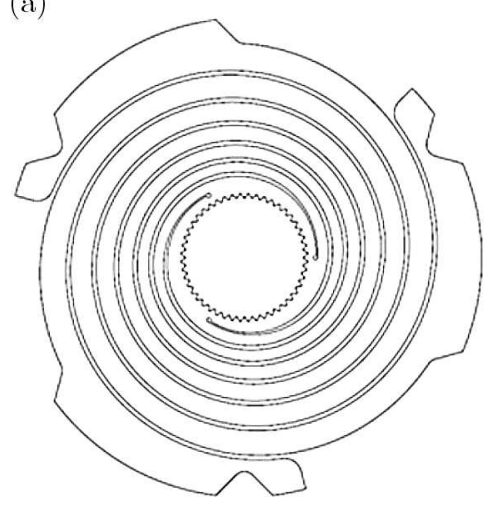

(b)

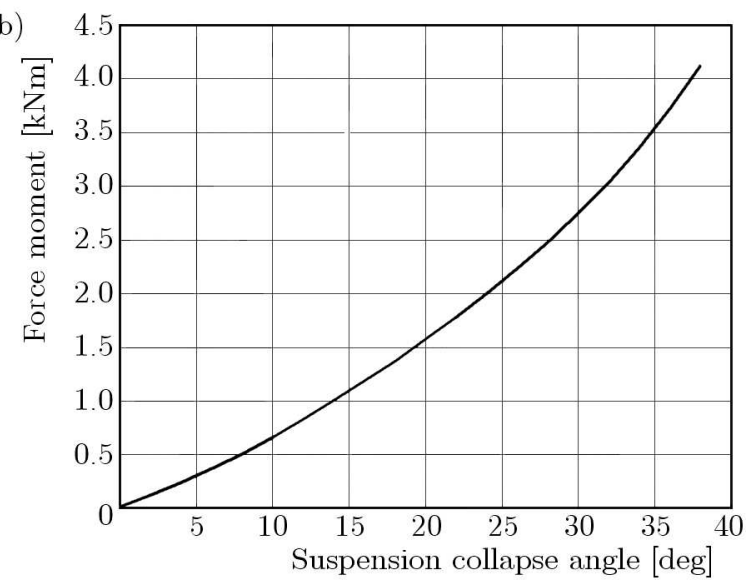

Fig. 6. Single logarithmic spring (a) and stiffness characteristics of the 15-spring packet (b)

\subsection{Basic model}

The suspension model of $2 \mathrm{~S} 1$ vehicle in the original version is equipped with torsional bars in the axles of all road wheels. The original hull mass in the model is assumed as $6.7 \mathrm{E}+03 \mathrm{~kg}$. The torsional stiffness of bars is assumed as linear, with coefficient $1.11 \mathrm{E}+02 \mathrm{Nm} / \mathrm{deg}$. All wheels in the models are numbered from the front of the vehicle to its end as presented on the basic model in Fig. 7. This is the basic model of $2 \mathrm{~S} 1$ vehicle in its initial equilibrium state from which the simulation of dynamics is started.

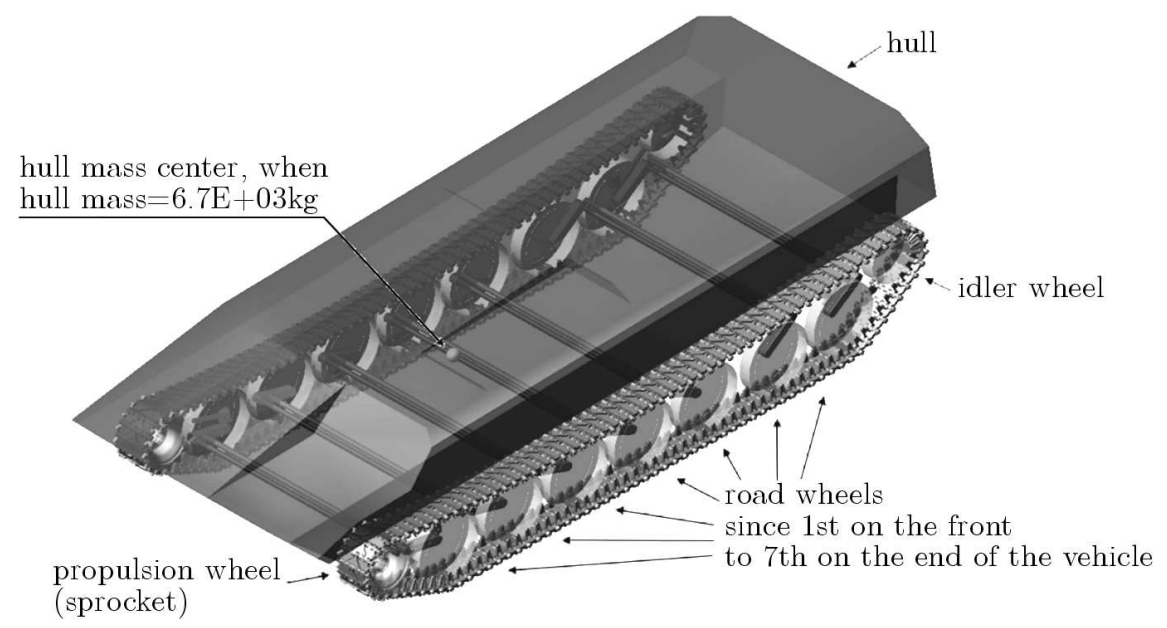

Fig. 7. Basic model of the completely tracked vehicle

\subsection{The first modernized model}

In this model, two torsional bars are removed, and in their place spiral torsional springs are inserted (Fig. 8). The torsional spring models are based on stiffness characteristics of the real logarithmic springs packet. As has been said before, this is a packet of 15 single springs connected together in a parallel system. In the real vehicle, such spring pockets work in various road conditions.

For this reason, the authors made simplification assumptions such as constant temperature range and linear characteristics in whole range of the collapse rebound movement. In the springs packet model, a potential reaction between the adjacent single springs and also between parts of the same spring are omitted. In the modernized vehicle model, the mass of the hull is increased 


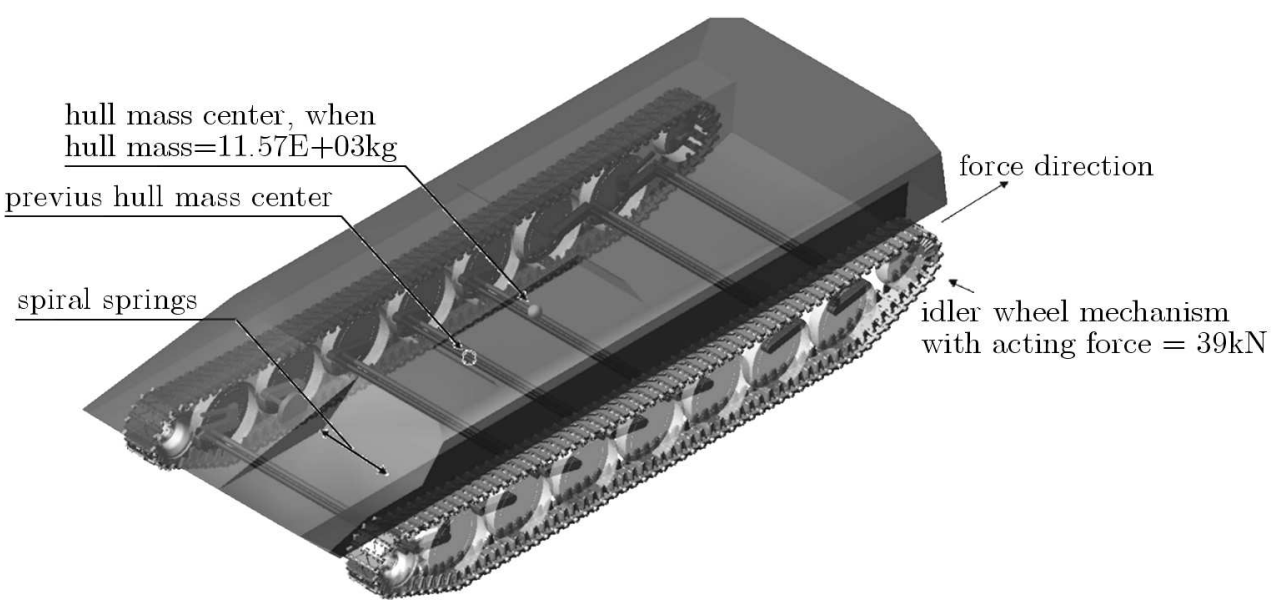

Fig. 8. Modernized model of the completely tracked vehicle

by about $4.87 \mathrm{E}+03 \mathrm{~kg}$ up to $11.57 \mathrm{E}+03 \mathrm{~kg}$. Also the hull mass center is moved closer to the geometrical hull center (see Fig. 8). The last modernization is associated with the idler mechanism. This mechanism acts on the idler wheel in the longitudinal axle of the vehicle with a constant force value, which equals $39 \mathrm{kN}$. This solution is used for maintaining a constant tension of the tracks. It is a simplified version of the real idler wheel mechanism which is based on an eccentric mechanism. However, this simplification realizes the main idea of the constant track tension concept.

\subsection{The second modernized model}

This model has the same parameters like the first modernized model, but the spring modules consisting of spiral springs are mounted on all road wheel axles. It is used for the estimation of the logarithmic spring effectiveness through comparison of the basic model with the second modernized model. Therefore, the stiffness in all axles increases, but the rest of parameters is the same like in the previous modernized model. Such a structural solution gives more free space inside the hull, which could be used for installing better floor-armour or other equipment. This solution improves also safety, because all reactive elements of the suspension system are removed from the floor plate to both sides, where they may be better covered.

\section{Simulation of dynamics of the platform models}

During investigation, all the mentioned models are simulated in conditions of the straight line driving on a flat road with a single obstacle. The obstacle has a triangular shape with length of the base $142 \mathrm{~mm}$ and height $71 \mathrm{~mm}$ (Fig. 9a). This obstacle is overcome only with the right side track (Fig. 9b).

When the obstacle is crossed, the model moves with a constant velocity equal to $3.8 \mathrm{~km} / \mathrm{h}$. The same velocity has been used in proving ground tests on a real 2S1 vehicle with parameters of the basic model. In the first phase of movement, the vehicle accelerates. It takes 8 seconds, but after the acceleration the vehicle starts to move with a constant velocity. In this quasi steady state, the vehicle crosses the obstacle. The first road wheel reaches the obstacle after 20 seconds from the simulation starting moment. Then it may be assumed that thewhole vehicle is in the above mentioned state because all acceleration effects should be suppressed. 
(a)

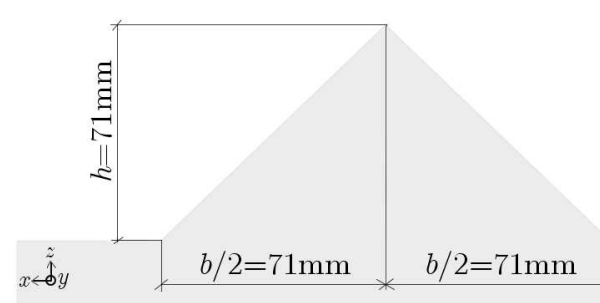

(b)

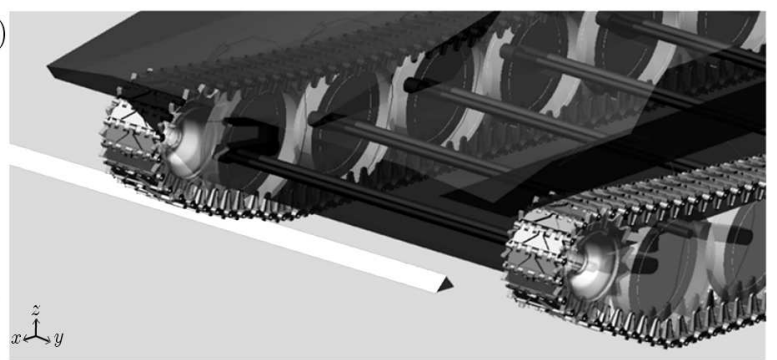

Fig. 9. Single obstacle: (a) dimensions, (b) view during simulation

\section{Analysis of the results}

In this Section, two types of results are presented. The first one is associated with time domain analysis, the second one with the frequency domain. Only analysis in these both domains can give full the view on the model. It shows not only its behaviour in time but also which frequency may be transmitted from the road wheels to the crew.

\subsection{Time domain analysis}

Results from the three above described models are compared during their analysis. In this Subsection, the authors present time histories of vertical acceleration of the vehicle hull mass center (Fig. 10a). The smallest amplitudes of acceleration are observed in the case of the first modified model. Such a situation is associated with bigger mass of the vehicle hull. In the case of the second modified model, where all axles are equipped with the logarithmic springs, the amplitudes of acceleration are higher, but not as high as in the basic model case. This higher amplitude of the vertical acceleration could be caused by higher stiffness of the spiral spring packets, in comparison to the stiffness of the torsional bars. In the case of the hull mass vertical acceleration, the difference between the basic model and the second modified model is not so big. In this situation, another indicator is more helpful. As it is known, for efficiency of the vehicle crew, comfort is very important. When the crew is exposed to activity of too high vibration energy, their efficiency is getting lower and their reaction time increases radically. To estimate the influence of energy on the human body, the authors used the Human Response Filter (HRF) developed by U.S. Army TACOM (Lee and Lins, 1973). The evaluation of the level of the absorbed power by the human body is possible by making use of this filter. Based on it, the authors calculated time histories of the Relative Cumulative Absorbed Power (RCAP), (see Fig. 10b). All results are relative to the power absorbed by the human body placed in the center of the hull mass in the basic model

$$
\mathrm{CAP}=\frac{1}{T} \int_{0}^{T} P d t \quad \mathrm{RCAP}=\frac{\mathrm{CAP}}{\mathrm{CAP}_{\max }}
$$

where $T$ is the time of power absorption, $P$ is the absorbed power in the vertical direction calculated from the vehicle hull vertical acceleration filtered by the HRF. The RCAP for the above three models are shown in Fig. 10b.

These time histories clearly show that the absorbed power in the case of both modified vehicle models are much lower than in the basic model. Therefore, in this situation, solutions of the modified models give noticeably better results than the basic model solution. In Fig. 10c, the pitch velocity is presented. This rotational velocity behaves similarly for all three models, and this effect could be neglected because the maximum level of this velocity is about $3 \mathrm{deg} / \mathrm{s}$. The last part of this figure (Fig. 10d) contains a comparison of the roll velocity of the vehicle 

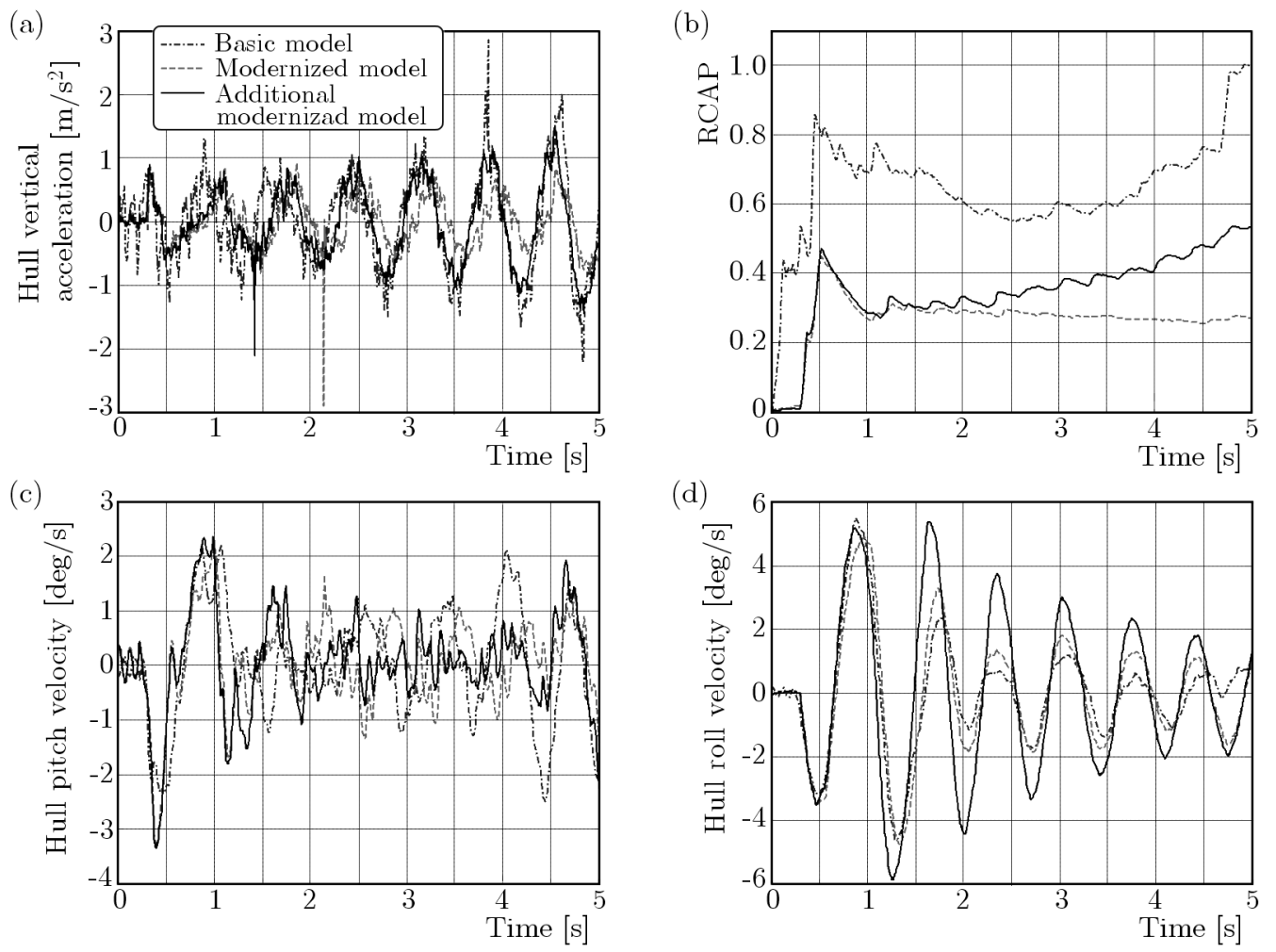

Fig. 10. Time histories of vertical acceleration of the vehicle hull (a), human body cumulative absorbed power (b), hull pitch angular velocity (c), hull roll angular velocity (d)

hull. In this case, the situation is different. The most stable model is the basic model, and the worst is the second modified model. The above mentioned figure contains the results for models with the so called average damping characteristics (see Fig. 5).

\subsection{Frequency domain analysis}

For a complete picture of the simulations, the results from the frequency domain analysis are shown. In Fig. 11a, one can see that in the basic model the main transmitted low frequency of the hull vertical acceleration is about $1 \mathrm{~Hz}$, while in the modified models, it is enclosed in the range from $2 \mathrm{~Hz}$ to $2.5 \mathrm{~Hz}$. The models transmit the similar low frequencies in the case of hull pitch and hull roll angular velocities.

The difference between the transmitted frequencies is mainly associated with the hull mass difference in the basic model and the modernized models.

\section{Conclusion}

In conclusion of analysis of the results, one can notice advantages of application of logarithmic torsional springs. Because of the higher stiffness level of the torsional springs packets than in the traditional bars, the designers can increase the vehicle hull mass. This enables one to resign from the axial offsets of the wheels, so the right and left wheels may be mounted on the same axles. Usage of the spiral spring packets gives more space between the wheels. This space may be used for additional equipment or even additional armour. Spiral springs increase also the safety level, because torsional bars are associated with reactive elements of the suspension under the floor of the crew cabin. During explosion of a charge placed under the vehicle, these reactive elements could be harmful for the crew. The spiral spring packets are placed on both sides of the 

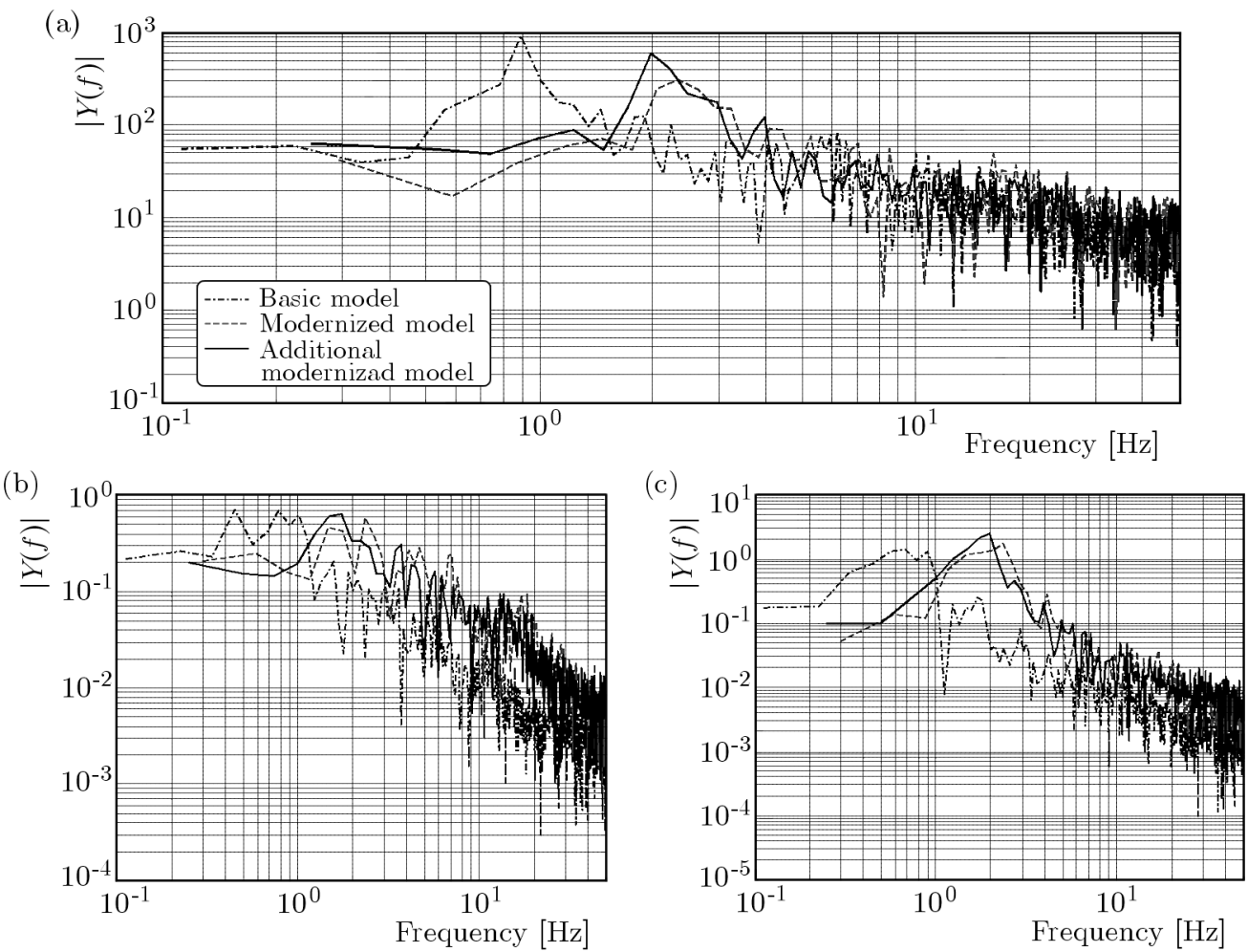

Fig. 11. Frequency characteristics of vertical acceleration of the vehicle hull (a), hull pitch angular velocity (b), hull roll angular velocity (c)

vehicle and could be covered. Also the RCAP indicator shows advantages of the application of logarithmic springs. It shows a significant reduction in the absorbed power by the crew bodies, which means a significant increase in the ride comfort. This feature of the suspension system increases the crew efficiency in the battlefield conditions.

\section{References}

1. ADAMS ver. 2010, Help Documentation (ADAMS/View, Solver), MSC, Software 2010

2. Donahue M.D., Hedrick J.K., 2003, Implementation of an Active Suspension, Preview Controller for Improved Ride Comfort, Nonlinear and Hybrid Systems in Automotive Control, Springer, 1-21, ISBN 978-1-85233-652-3

3. Huн K., Сно B.H., Chог J.H., 2011, Development of a track tension monitoring system in tracked vehicles on flat ground, Proceedings of the Institution of Mechanical Engineer, Part D: Journal of Automobile Engineering, 215, 567-578

4. Jurkiewicz A., Micek P., Aposto€ M., 2010, Laboratory investigation on ahydraulic force generator (in Polish), Hydraulika i Pneumatyka, 4, 29-32, ISSN 1505-3954

5. Kowal J., Jurkiewicz A., Aposto€ M., 2013, A study of a new suspension system of caterpillar platform (in Polish), Proceedings of 26th Scientific conference on Trends in the Development of Heavy Duty Machines, 64, ISBN 978-83-932590-4-5

6. Kubela T., Pochyly A., Singule V., 2010, Advanced tools for multi-body simulation and design of control structures applied in robotic system development, Solid State Phenomena, 164, 387-391

7. LEE K., 2000, A numerical method for dynamic analysis of tracked vehicles of high mobility, KSME International Journal, 14, 10, 1028-1040 
8. LeE R.A., Lins W.F., 1973, Human Vibration Measuring Instrument, Technical Report No. AD785648, U.S. Army Tank-Automotive Command, Warren, Michigan

9. ŁukASzEWICZ A., 2009, Modelling of solid part using multibody techniques in parametric CAD systems, Solid State Phenomena, 147/149, 924-929

10. Nabageo T., Jurkiewicz A., Apostoe M., Micek P., 2011, Construction and simulation of a 2S1 tracked vehicle model and its verification using vertical forces on the road wheels while overcoming a single obstacle, Solid State Phenomena, 177, 168-176.

11. Nabageo T., Jurkiewicz A., Apostoє M., Micek P., 2014, Simulation of 2S1 tracked vehicle model with modernized suspension system during crossing a single obstacle, Solid State Phenomena, 208, 140-147

12. Ryu H.S., Bae D.S., Choi J.H., Shabana A.A., 2000, A compliant track link model for high-speed, high-mobility tracked vehicles, International Journal for Numerical Methods in Engineering, 48,1481-1502

Manuscript received July 22, 2013; accepted for print March 14, 2014 